\section{On Optimal Analysis/Synthesis Filters for Coding Gain Maximization}

Igor Djokovic and P. P. Vaidyanathan

Abstract-We consider the use of pre and postfilters in conjunction with $M$-channel, uniform-band paraunitary (orthonormal) filter banks. We show that given any orthonormal filter bank, the pre and postfilters that maximize the coding gain are determined entirely by the power spectrum of the input process regardless of the details of the orthonormal filter bank (which could be FIR, IIR, or even the ideal brickwall filter bank). The optimized coding gain, however, depends on the prefilter as well as the sandwiched orthonormal filter bank. The coding gain improvement due to pre and postfiltering is often significant as we demonstrate with numerical examples and comparisons. The validity of our results depends strongly on the orthonormality property of the filter bank in between the pre and postfilters. In the nonorthonormal case, most of these results are not true, as is demonstrated.

\section{INTRODUCTION}

A typical filter bank (FB) used for subband coding is shown in Fig. 1. The input signal $x(n)$ (assumed a discrete time wide sense stationary (WSS) random process) is passed through the analysis filters $H_{k}(z)$. Subband signals $x_{k}(n)$ are quantized, and then transmitted or stored. The performance of a subband coding system is often expressed in terms of its coding gain, defined below.

Definition 1.1: Let the noise variance of a PCM coder be $\sigma_{P C M}^{2}$, and the averaged noise variance of a subband coding system be $\sigma_{S B C}^{2}$. Under the constraint of equal total number of bits per sample, the coding gain is defined as the ratio $G=\sigma_{P C M}^{2} / \sigma_{S B C}^{2}$.

Several aspects of the coding gain optimization and its connection to the so-called energy compaction problem have been addressed by a number of authors in the recent literature [1]--[5]. In this correspondence, we prove a very specific result that pertains to the system shown in Fig. 2. This is a special case of Fig. 1, with $H_{k}(z)=P(z) P_{k}(z)$ and $F_{k}(z)=Q_{k}(z) / P(z)$. Given that the "sandwiched filter bank" system $\left\{P_{k}(z), Q_{k}(z)\right\}$ is paraunitary (PU) or orthonormal [6], we show that the best prefilter $P(z)$ that maximizes the coding gain is such that $\left|P\left(e^{j w}\right)\right|=\left[1 / S\left(e^{j w}\right)\right]^{1 / 4}$ (the phase of $P(z)$ does not matter). Thus, the solution is independent of the details of the sandwiched PU system $\left\{P_{k}(z) . Q_{k}(z)\right\}$, though the coding itself depends on both $P(z)$ and $\left\{P_{k}(z), Q_{k}(z)\right\}$. While this solution resembles the half-whitening result known to lossy data compression experts [7], it should not be regarded as an obvious application of that result. In fact, the result does not extend to the case where the sandwiched filter bank $\left\{P_{k}(z), Q_{k}(z)\right\}$ is not orthonormal, as we shall show in Section II.

\section{Coding Gain Optimization}

In order to derive an expression for the coding gain of a biorthogonal $\mathrm{FB}$, the noise sources produced by the subband quantizers are assumed white and uncorrelated. This is a reasonable assumption as long as the subband signals are not too coarsely quantized.

Manuscript received November 28, 1993; revised July 11, 1995. This work was supported by the National Science Foundation under Grant MIP 9215785 , by Tektronix, Inc., and by Rockwell International. The associate editor coordinating the review of this work and approving it for publication was Prof. Banu Onaral.

The authors are with the Department of Electrical Engineering, California Institute of Technology, Pasadena, CA 91125 USA.

Publisher Item Identifier S 1053-587X(96)03061-9.

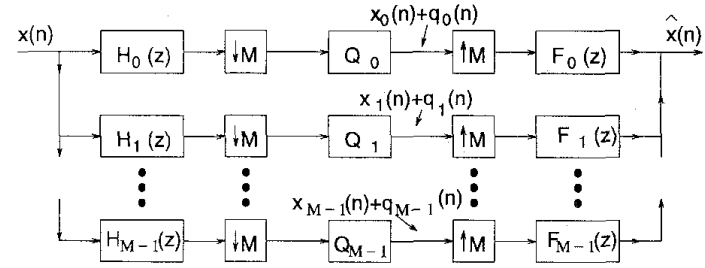

Fig. 1. Uniform filter bank used for subband coding.

\section{A. Coding Gain of a Biorthogonal Filter Bank}

Consider a uniform perfect reconstruction (PR) FB as in Fig. 1. The variances of the subband signals $x_{k}(n)$ are

$$
\begin{gathered}
\sigma_{x_{k}}^{2}=\frac{1}{2 \pi} \int_{-\pi}^{\pi} S\left(e^{j w}\right)\left|H_{k}\left(e^{j w}\right)\right|^{2} d w, \\
k=0,1 \cdots, M-1
\end{gathered}
$$

where $S\left(e^{j w}\right)$ is the power spectral density (PSD) function of the input random process. The noise PSD function at the output of the $k$ th quantizer (see [7]) is

$$
\begin{aligned}
S_{q_{k} q_{k}}\left(e^{j w}\right) & =E\left[q_{k}(n) q_{k}^{*}(n)\right] \\
& =C 2^{-2 b_{k}} \sigma_{x_{k}}^{2} \\
& =\sigma_{q k}^{2}
\end{aligned}
$$

where $b_{k}$ is the number of bits allocated to the $k$ th channel, $C$ is some constant that depends on the statistics of $x_{k}(n)$, and $q_{k}(n)$ is the noise sequence. After some WSS random process passes through an expander, it becomes a cyclo-WSS process (see [8]). The period of cyclostationarity is $M$. Then we can average the variance over the period to get $\sigma_{o u t, k}^{2}=\left(\sigma_{q k}^{2} / M\right) \|\left. f_{k}(n)\right|_{2} ^{2}$. The subband noises are uncorrelated and they remain such after passing through the synthesis filters. Then the output noise variance is $\sigma_{S B C}^{2}=\sum_{k=0}^{M-1} \sigma_{o u t}^{2}, k$. Using (2.2) and (2.1), we get

$$
\begin{aligned}
\sigma_{S B C}^{2}= & \frac{C}{M} \sum_{k=0}^{M-1} 2^{-2 b_{k}} \frac{1}{2 \pi} \int_{-\pi}^{\pi}\left|F_{k}\left(e^{j w}\right)\right|^{2} d w \\
& \cdot \frac{1}{2 \pi} \int_{-\pi}^{\pi} S\left(e^{j w}\right)\left|H_{k}\left(e^{j w}\right)\right|^{2} d w .
\end{aligned}
$$

The average bit rate is $b=1 / M \sum_{k=0}^{M-1} b_{k}$. If we quantized the input signal to this number of bits, without any subband decomposition, i.e., just PCM coding, the noise variance would be [7]

$$
\sigma_{P C M}^{2}=C 2^{-2 b} \sigma_{x}^{2}=C 2^{-2 b} \frac{1}{2 \pi} \int_{-\pi}^{\pi} S\left(e^{j w}\right) d w .
$$

So the coding gain, defined as the ratio of the above variances, is

$$
\begin{aligned}
G= & \frac{\sigma_{P C M}^{2}}{\sigma_{S B C}^{2}} \\
= & {\left[2^{-2 b} \frac{1}{2 \pi} \int_{-\pi}^{\pi} S\left(e^{j w}\right) d w\right] /\left[\frac{1}{M} \sum_{k=0}^{M-1} 2^{-2 b_{k}}\right.} \\
& \cdot \frac{1}{2 \pi} \int_{-\pi}^{\pi}\left|F_{k}\left(e^{j w}\right)\right|^{2} d w \\
& \left.\cdot \frac{1}{2 \pi} \int_{-\pi}^{\pi} S\left(e^{j w}\right)\left|H_{k}\left(e^{j w}\right)\right|^{2} d w\right]
\end{aligned}
$$

One of the optimization steps is an optimal bit allocation. We can make this step now and minimize the denominator. The optimal bit 


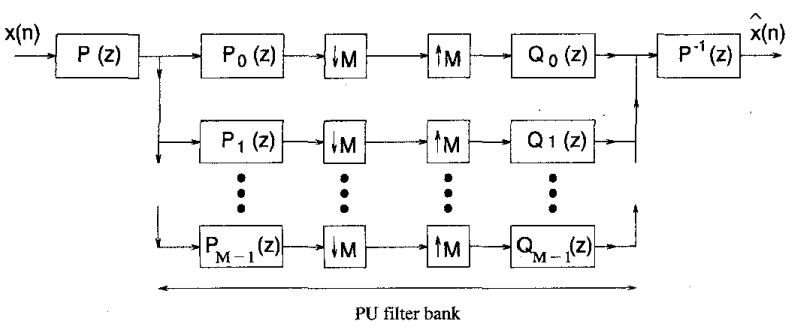

Fig. 2. Filter bank with pre and postfilters.

allocation (see [2]) turns the sum in the denominator into a product. So we have the following expression for the coding gain under optimal bit allocation.

$$
\begin{aligned}
G= & \frac{\sigma_{P C M}^{2}}{\sigma_{S B C}^{2}} \\
= & {\left[\frac{1}{2 \pi} \int_{-\pi}^{\pi} S\left(e^{j w}\right) d w\right] / } \\
& {\left[\left(\prod_{k=0}^{M-1} \frac{1}{2 \pi} \int_{-\pi}^{\pi}\left|F_{k}\left(e^{j w}\right)\right|^{2} d w\right.\right.} \\
& \left.\left.\cdot \frac{1}{2 \pi} \int_{-\pi}^{\pi} S\left(e^{j w}\right)\left|H_{k}\left(e^{j w}\right)\right|^{2} d w\right)^{1 / M}\right] .
\end{aligned}
$$

This coding gain formula is valid as long as the subband noises are white and uncorrelated.

\section{B. Prefilters for PU Filter Banks}

Consider the class of prefiltered paraunitary (PPU) filter banks obtained by putting pre and postfilters around a PU FB $\left\{P_{k}(z), Q_{k}(z)\right\}$ (see Fig. 2). The aim of this subsection is to find a PPU FB that maximizes coding gain.

First, notice that maximization of the coding gain is the same as minimization of the denominator of (2.5). From the Cauchy-Schwarz inequality it follows that

$$
\begin{gathered}
\frac{1}{2 \pi} \int_{-\pi}^{\pi}\left|F_{k}\left(e^{j w}\right)\right|^{2} d w \frac{1}{2 \pi} \int_{-\pi}^{\pi} S\left(e^{j w}\right)\left|H_{k}\left(e^{j w}\right)\right|^{2} d w \geq \\
{\left[\frac{1}{2 \pi} \int_{-\pi}^{\pi}\left|H_{k}\left(e^{j w}\right) F_{k}\left(e^{j w}\right)\right| \sqrt{S\left(e^{j w}\right)} d w\right]^{2}}
\end{gathered}
$$

with equality achieved if and only if

$$
\left|H_{k}\left(e^{j w}\right)\right| \sqrt{S\left(e^{j w}\right)}=\lambda_{k}\left|F_{k}\left(e^{j w}\right)\right| \text { for } 0 \leq k \leq M-1,
$$

for arbitrary choice of $\lambda_{k} \neq 0$ (e.g., $\lambda_{k}=1 \quad \forall k$ ). Notice that the PSD function is $S\left(e^{j w}\right) \geq 0$, so that its square root is well defined.

Since $H_{k}(z)=P_{k}(z) P(z)$ and $F_{k}(z)=Q_{k}(z) / P(z)$, we have $H_{k}(z) F_{k}(z)=P_{k}(z) Q_{k}(z)$. So the right-hand side in (2.6) depends only on the product $P_{k}(z) Q_{k}(z)$, and is independent of the prefilter $P(z)$. Thus, if the prefilter $P(z)$ can be chosen to achieve equality in (2.6) for all $k$, it will maximize the coding gain for a fixed filter bank $\left\{P_{k}(z), Q_{k}(z)\right\}$. This observation is true whether the sandwiched system $\left\{P_{k}(z), Q_{k}(z)\right\}$ is orthonormal or biorthogonal. However, when $\left\{P_{k}(z), Q_{k}(z)\right\}$ is orthonormal, equality in (2.6) is achievable for all $k$. To see this, note that $(2.7)$ can be rewritten as

$$
\left|P\left(e^{j w}\right) P_{k}\left(e^{j w}\right)\right| \sqrt{S\left(e^{j w}\right)}=\left|\frac{1}{P\left(e^{j w}\right)} Q_{k}\left(e^{j w}\right)\right| .
$$

When $\left\{P_{k}(z), Q_{k}(z)\right\}$ is an orthonormal filter bank, then $Q_{k}\left(e^{j w}\right)=P_{k}^{*}\left(e^{j w}\right)$ for perfect reconstruction [6]. Therefore, the condition (2.8) for equality reduces to

$$
\left|P\left(e^{j w}\right)\right|=\left[\frac{1}{S\left(e^{j w}\right)}\right]^{1 / 4}
$$

and is independent of $k$. The coding gain (2.5) becomes

$$
G_{P P U}=\frac{\frac{1}{2 \pi} \int_{-\pi}^{\pi} S\left(e^{j w}\right) d w}{\left[\prod_{k=0}^{M-1} \frac{1}{2 \pi} \int_{-\pi}^{\pi}\left|P_{k}\left(e^{j w}\right)\right|^{2} \sqrt{S\left(e^{j w}\right)} d w\right]^{2 / M}} .
$$

Summarizing, we have proved the following theorem.

Theorem 2.1: Consider the class of all PR FB's that can be obtained from the structure in Fig. 2, where $\left\{P_{k}(z), Q_{k}(z)\right\}$ is a PU FB. Then, the prefilter that maximizes coding gain will satisfy (2.9).

Summarizing our main point, if we wish to find an optimal filter bank of the form in Fig. 2, where $\left\{P_{k}(z), Q_{k}(z)\right\}$ is orthonormal, we construct $P\left(e^{j w}\right)$ according to (2.9), and construct $\left\{P_{k}(z), Q_{k}(z)\right\}$ to be the orthonormal filter bank that maximizes the coding gain for an input with power spectrum $\sqrt{S\left(e^{j w}\right)}$. Then the coding gain with the optimal prefilter is given by (2.10). The orthonormal filter bank that maximizes this coding gain is the one that has maximum coding gain for an input with the power spectrum $\sqrt{S\left(e^{j w}\right)}$. There are techniques to identify such a system based on the work by Unser [3]; also, see [9]. We shall not go into details of this here. From the above, we see that the optimization of $P(z)$ has been decoupled from that of $\left\{P_{k}(z), Q_{k}(z)\right\}$. This establishes the following corollary.

Corollary 2.1: Putting pre and postfilters as given by (2.9) around any PU FB $\left\{P_{k}(z), Q_{k}(z)\right\}$ will not decrease its coding gain. It will strictly increase the coding gain if the input spectrum is not piecewise constant.

An insightful way to understand the above corollary is as follows. If we put $F_{k}\left(e^{j w}\right)=\tilde{P}_{k}\left(e^{j w}\right)$ and $H_{k}\left(e^{j w}\right)=P_{k}\left(e^{j w}\right)$ into (2.5), and use the fact that PU filters have unit energy, then after optimal bit allocation we get the coding gain of $\left\{P_{k}(z), Q_{k}(z)\right\}$ with input $x(n)$

$$
G_{P U}=\frac{\frac{1}{2 \pi} \int_{-\pi}^{\pi} S\left(e^{j w}\right) d w}{\left[\prod_{k=0}^{M-1} \frac{1}{2 \pi} \int_{-\pi}^{\pi}\left|P_{k}\left(e^{j w}\right)\right|^{2} S\left(e^{j w}\right) d w\right]^{1 / M}} .
$$

The ratio of the two coding gains

$$
\begin{aligned}
\eta & =\frac{G_{P P U}}{G_{P U}} \\
& =\prod_{k=0}^{M-1}\left\{\frac{\frac{1}{2 \pi} \int_{-\pi}^{\pi}\left|P_{k}\left(e^{j w}\right)\right|^{2} S\left(e^{j w}\right) d w}{\left[\frac{1}{2 \pi} \int_{-\pi}^{\pi}\left|P_{k}\left(e^{j w}\right)\right|^{2} \sqrt{S\left(e^{j w}\right)} d w\right]^{2}}\right\}^{1 / M}
\end{aligned}
$$

satisfies $\eta \geq 1$. This is because for each $k$, we have

$$
\begin{aligned}
& {\left[\frac{1}{2 \pi} \int_{-\pi}^{\pi}\left|P_{k}\left(e^{j w}\right)\right|^{2} \sqrt{S\left(e^{j w}\right)} d w\right]^{2}} \\
& \quad \leq \frac{1}{2 \pi} \int_{-\pi}^{\pi}\left|P_{k}\left(e^{j w}\right)\right|^{2} S\left(e^{j w}\right) d w
\end{aligned}
$$

with equality if and only if $S\left(e^{j w}\right)$ is a constant over the support of $P_{k}\left(e^{j w}\right)$. This follows from the Cauchy-Schwarz inequality, and the fact that the energy of $P\left(e^{j w}\right)$ is unity. We see that this simple 
system always outperforms any PU system, as long as $S\left(e^{j w}\right)$ is not constant where $P_{k}\left(e^{j w}\right) \neq 0$. This improvement in the performance is more significant as $S\left(e^{j w}\right)$ has more nonconstant behavior.

Relation to Half-Whitening: A well-known data compression technique called half-whitening is described in [7]. Here, a signal $x(n)$ is first prefiltered with a filter $H(z)$, then quantized and postfiltered with $1 / H(z)$. Under mild assumptions on the joint statistics of the signal $x(n)$ and the quantizer noise, the best prefilter (to maximize the output signal to noise ratio) is such that $\left|H\left(e^{j w}\right)\right|=\left[1 / S\left(e^{j w}\right)\right]^{1 / 4}$. Our result in Theorem 2.1 shows that a similar result is true if the quantizer is replaced with a paraunitary subband coder. If the filter bank $\left\{P_{k}(z), Q_{k}(z)\right\}$ is not orthonormal, then the preceding results are not true. For example, if $\left\{P_{k}(z), Q_{k}(z)\right\}$ were biorthogonal rather than orthonormal, then the insertion of $P(z)$ and $1 / P(z)$ with $P(z)$ as in (2.9) could even decrease the coding gain. Here is a way to visualize such a situation: suppose $\left\{P_{k}(z), Q_{k}(z)\right\}$ is itself a biorthogonal filter bank obtained by sandwiching an orthonormal filter bank between an optimal prefilter (2.9) and a postfilter. If we now insert another pair of $P(z)$ and $1 / P(z)$ (with $P(z)$ still given as in (2.9)), it can only decrease the coding gain! Theorem 2.1 and Corollary 2.1 should not, therefore be regarded as a simple extension of the half-whitening result.

Relation to Prediction Gain: In order to better understand why this scheme works, let us look at the following expression:

$$
\left\{\prod_{k=0}^{M-1}\left[\frac{1}{2 \pi} \int_{-\pi}^{\pi}\left|P_{k}\left(e^{j w}\right)\right|^{2} S^{p}\left(e^{j w}\right) d w\right]^{1 / p}\right\}^{1 / M} .
$$

Notice that when $p=1$, this is the denominator in (2.11) (the case of PU FB); and when $p=1 / 2$, it is the denominator in (2.10) (the case of PPU). Now consider the theoretical bound on the coding gain, namely the prediction gain.

$$
G_{\infty}=\frac{\sigma_{x}^{2}}{\exp \left\{\frac{1}{2 \pi} \int_{-\pi}^{\pi} \log _{e}\left[S\left(e^{j w}\right)\right] d w\right\}} .
$$

The denominator here can be obtained from the expression (2.14) as the limit when $p \rightarrow 0$. For this, note that $1 /(2 \pi) \int\left|P_{k}\left(e^{j w}\right)\right|^{2} d w=$ 1 and $\sum_{k=0}^{M-1}\left|P_{k}\left(e^{j w}\right)\right|^{2}=M$, since $\left\{P_{k}(z), Q_{k}(z)\right\}$ is a PU FB. Then (see [10]) we get

$$
\begin{aligned}
& \lim _{p \rightarrow 0}\left\{\prod_{k=0}^{M-1}\left[\frac{1}{2 \pi} \int_{-\pi}^{\pi}\left|P_{k}\left(e^{j w}\right)\right|^{2} S^{p}\left(e^{j w}\right) d w\right]^{1 / p}\right\}^{1 / M} \\
& =\left(\prod_{k=0}^{M-1} \exp \left\{\frac{1}{2 \pi} \int_{-\pi}^{\pi}\left|P_{k}\left(e^{j w}\right)\right|^{2} \log _{e}\left[S\left(e^{j w}\right)\right] d w\right\}\right)^{1 / M} \\
& =\exp \{\frac{1}{2 \pi} \int_{-\pi}^{\pi} \underbrace{\frac{\sum_{k=0}^{M-1}\left|P_{k}\left(e^{j w}\right)\right|^{2}}{M}}_{=1} \log _{e}\left[S\left(e^{j w}\right)\right] d w\} \\
& =\exp \left\{\frac{1}{2 \pi} \int_{-\pi}^{\pi} \log _{e}\left[S\left(e^{j w}\right)\right] d w\right\} .
\end{aligned}
$$

Therefore, we improved the coding gain of a PU system (which corresponds to $p=1$ ) by finding the structure in which $p=1 / 2$. If there existed a structure corresponding to $p<1 / 2$, it would further improve the coding gain. ${ }^{1}$ The examples below will demonstrate that our technique approximately halves the gap between the performance of a PU system and the prediction gain bound (2.15) on a decibel scale.

${ }^{1}$ It can be shown using Jensen's inequality (see [10]) that, as $p$ decreases, the coding gain can only increase.

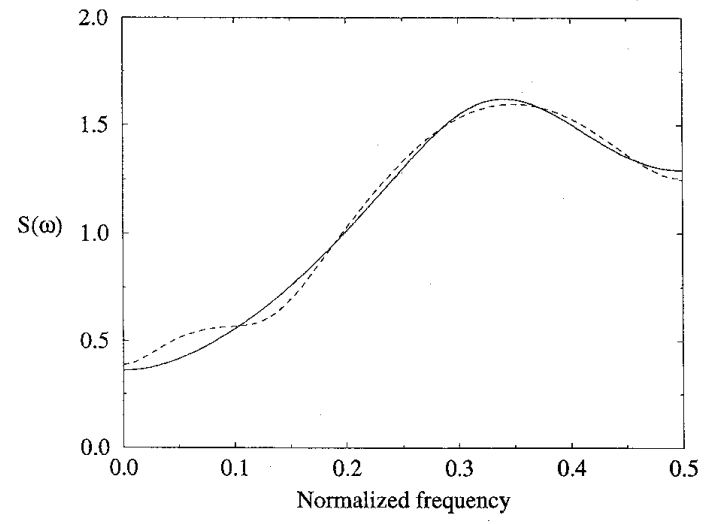

Fig. 3. Plots of $S^{-1 / 4}\left(e^{j w}\right)$ for a test example (dotted curve) and a rational approximation $\left|P_{n}\left(e^{j w}\right)\right|$ (solid curve). The approximation filter $P_{a}\left(e^{j w}\right)$ is a second-order IIR filter.

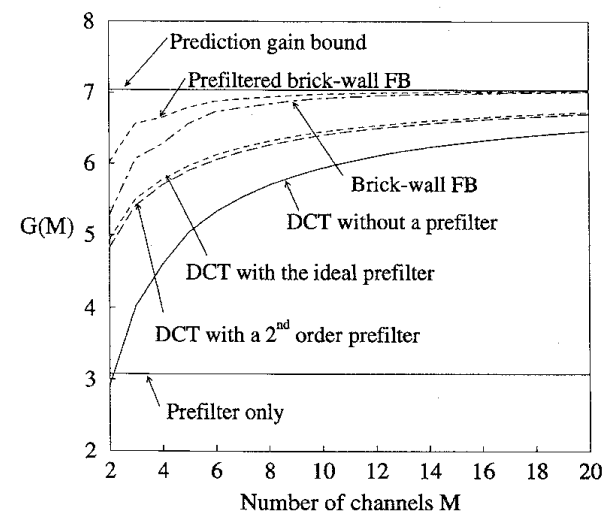

Fig. 4. Example 3.1. Coding gain of DCT filter banks as a function of the number of channels, with and without prefilters.

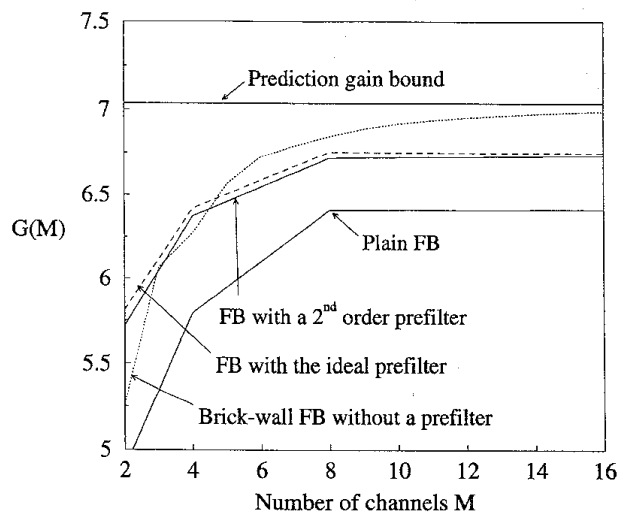

Fig. 5. Example 3.2. Coding gain of tree-structured filter banks as a function of the number of channels, with and without prefilters.

Realizability: In practice, we have to approximate $P\left(e^{j w}\right)$ and $1 / P\left(e^{j w}\right)$ with rational filters. The phase of pre and postfilters does not matter, but stability does. As long as $S\left(e^{j w}\right)$ is bounded, we can find a good (stable) approximation of $S^{1 / 4}\left(e^{j w}\right)$, and use it as the postfilter $1 / P\left(e^{j w}\right)$. In order to ensure stability of the prefilter $P\left(e^{j w}\right)$, we have to make sure that $1 / P\left(e^{j w}\right)$ is a minimum phase 
approximation of $S^{1 / 4}\left(e^{j w}\right)$ (one obvious way of doing this is by autoregresive (AR) modeling of $S^{1 / 4}\left(e^{j w}\right)$ ). If $S\left(e^{j w}\right)=0$ on some interval, it can be shown that both pre and postfilters can be chosen to be zero on the same interval, so that there are no stability problems. The AR modeling approach not only insures stability of the pre and postfilters, but it also offers a computationally very efficient way of obtaining rational approximations of optimal pre and postfilters. In order to obtain a minimum phase stable approximation of $S^{-1 / 4}\left(e^{j w}\right)$, all we have to do is compute $\sqrt{S\left(e^{j w}\right)}$ (using the fast Fourier transform, for example), and then use Levinson's recursion to find a polynomial approximation of $S^{-1 / 4}\left(e^{j w}\right)$.

\section{EXAMPLES}

Example 3.1-DCT Filter Bank with Prefiltering: The above developed technique will be applied to a very simple PU FB. Let $\left\{P_{k}(z), Q_{k}(z)\right\}$ be a DCT FB, i.e., the one in which the polyphase matrix $\mathrm{E}(z)$ is the DCT IV matrix [11]. The DCT filters have poor attenuations. Fig. 3 shows $\left[1 / S\left(e^{j w}\right)\right]^{1 / 4}$, the test function chosen for this example (dotted curve). The solid curve is its second-order rational approximation (i.e., $P_{a}(z)$ is a second-order filter). The input PSD function $S\left(e^{j w}\right)$ was the lowpass $\mathrm{AR}(5)$ model of speech [7]. Fig. 4 shows the coding gain for different FB's. We can see that even prefilter alone (without any $\mathrm{FB}$ ) gives some coding gain (see [7], Ch. 7). The coding gain changes only slightly if the ideal prefilter $\left[1 / S\left(e^{j w}\right)\right]^{1 / 4}$ is approximated by a second-order rational filter. Notice that the coding gain of PPU FB approximately halves the gap (on a $\mathrm{dB}$ scale) between the coding gain achieved with the PU FB and the prediction gain bound on the coding gain given by (2.15). The next example is striking in the sense that a finite-order FB performs better than a brick-wall FB.

Example 3.2-Tree-Structured Filter Bank with Prefiltering: In the previous example, the DCT filters had poor frequency responses. In this example, we design tree structured FB's (number of channels $M$ is a power of 2) using a two-channel PU FB as a basic building block. The filter length of each filter in the two-channel module is eight (8A from [12]). We use the same second-order approximation of $\left[1 / S\left(e^{j w}\right)\right]^{1 / 4}$ as in Fig. 3. Now we can see in Fig. 5 that the PPU finite order FB's (for $M=2,4$ ) perform better than the corresponding ideal brick-wall FB's, which shows that an ideal brickwall FB does not necessarily maximize the coding gain for a given number of channels.

\section{CONCLUSION}

We showed how to optimally design the filters in a prefiltered paraunitary filter bank. It was shown that the coding gain of any PU FB can be improved by prefiltering. The choice of the prefilter and PU FB were shown to be independent of each other, depending only on the input PSD. The theory was demonstrated on two simple examples. The problem of finding the optimal FB over the class of all biorthogonal FB's is still open.

\section{REFERENCES}

[1] A. N. Akansu and Y. Liu, "On signal decomposition techniques," Opt. Eng., vol. 30, pp. 912-920, July 1991.

[2] A. Soman and P. P. Vaidyanathan, "Coding gain in paraunitary analysis/synthesis systems," IEEE Trans. Signal Processing, vol. 41, pp. 1824-1836, May 1993.
[3] M. Unser, "On the optimality of ideal filters for pyramid and wavelet signal approximation," IEEE Trans. Signal Processing, vol. 41, no. 12, pp. 3591-3596, Dec. 1993.

[4] M. Goldburg, "Applications of wavelets to quantization and random process representations," Ph.D. dissertation, Stanford Univ., Stanford, CA, May 1993.

[5] P. H. Westernik, J. Biemond, and D. E. Boekee, "An optimal allocation algorithm for subband coding," in Proc. IEEE Int. Conf. Acoust., Speech Signal Processing, New York, Apr. 1988, pp. 757-760.

[6] P. P. Vaidyanathan, Multirate Systems and Filter Banks. Englewood Hills, NJ: Prentice-Hall, 1993.

[7] N. S. Jayant and P. Noll, Digital Coding of Waveforms: Principles and Applications to Speech and Video. Englewood Cliffs, NJ: Prentice-Hall Signal Processing Series, 1984.

[8] V.P. Sathe and P. P. Vaidyanathan, "Effects of multirate systems on the statistical properties of random signals," IEEE Trans. Signal Processing, vol. 41, pp. 131-146, Jan. 1993.

[9] M. K. Tsatsanis and G. B. Giannakis, "Principal component filter banks for optimum wavelet analysis," in Proc, 6th Signal Processing Workshop Stat. Signal Array Processing, Victoria, Canada, Oct. 1992, pp. 50-53.

[10] W. Rudin, Real and Complex Analysis, 3rd ed. New York: McGrawHill, 1987.

[11] P. Yip and K. R. Rao, "Fast discrete transforms," in Handbook of Digital Signal Processing, D. F. Elliott, Ed. San Diego, CA: Academic, 1987.

[12] P. P. Vaidyanathan and P.-Q. Hoang, "Lattice structures for optimal design and robust implementation of two-channel perfect reconstruction QMF banks," IEEE Trans. Acoust., Speech, Signal Processing, vol. 36, pp. $81-94,1988$.

\section{A $B$-Wavelet-Based Noise-Reduction Algorithm}

Phillip L. Ainsleigh and Charles K. Chui

Abstract-A wavelet-based method is introduced for removing structured noise (e.g., impulsive spikes or unwanted harmonic components) from data. For this type of noise, the time- and frequency-localization capabilities of wavelets provide better noise detection and less signal distortion than direct filtering of data. The procedure is applied to timeseries data with impulsive noise and transfer-function data with multipath interference.

\section{INTRODUCTION}

The desire to eliminate structured noise from data arises in a variety of signal processing and statistical applications. For example, noise-reduction methods can be used to remove impulsive noise from musical or image data [1], and to remove unwanted harmonic components such as multipath interference in transfer function measurements [2] or seasonal variations in econometric trend analyses [3]. In these examples, parametric estimation cannot be used either for lack of an a priori model or because parametric modeling would constrain the analysis in an undesirable way. Previously, moving average and median filters have been applied to noisy data (e.g.,

Manuscript received March 18, 1994; revised October 10, 1995. This work was supported by the Office of Naval Research, the Army Research Office, and the National Science Foundation. The associate editor coordinating the review of this paper and approving it for publication was Prof. Banu Onaral.

P. L. Ainsleigh is with the Naval Undersea Warfare Center, Underwater Sound Reference Detachment, Orlando, FL 32856 USA (e-mail: painsleigh@usrd.nuwc.navy.mil).

C. K. Chui is with the Center for Approximation Theory, Texas A\&M University, College Station, TX 77843 USA (e-mail: cchui@math.tamu.edu).

Publisher Item Identifier S 1053-587X(96)03053-X. 